\title{
Numerical Study of Viscoelastic Micropolar Heat Transfer from a Vertical Cone for Thermal Polymer Coating
}

https://doi.org/10.1515/nleng-2018-0064

Received March 29, 2018; revised May 26, 2018; accepted August 3 , 2018.

\begin{abstract}
A mathematical model is developed to study laminar, nonlinear, non-isothermal, steady-state free convection boundary layer flow and heat transfer of a micropolar viscoelastic fluid from a vertical isothermal cone. The Eringen model and Jeffery's viscoelastic model are combined to simulate the non-Newtonian characteristics of polymers, which constitutes a novelty of the present work. The transformed conservation equations for linear momentum, angular momentum and energy are solved numerically under physically viable boundary conditions using a finite difference scheme (Keller Box method). The effects of Deborah number (De), Eringen vortex viscosity parameter $(\mathrm{R})$, ratio of relaxation to retardation times $(\lambda)$, micro-inertia density parameter (B), Prandtl number (Pr) and dimensionless stream wise coordinate $(\xi)$ on velocity, surface temperature and angular velocity in the boundary layer regime are evaluated. The computations show that with greater ratio of retardation to relaxation times, the linear and angular velocity are enhanced whereas temperature (and also thermal boundary layer thickness) is reduced. Greater values of the Eringen parameter decelerate both the linear velocity and micro-rotation values and enhance temperatures. Increasing Deborah number decelerates the linear flow and Nusselt number whereas it increases temperatures and boosts micro-rotation magnitudes. The study is relevant to non-Newtonian polymeric thermal coating processes.
\end{abstract}

Keywords: Jeffrey's viscoelastic model, Eringen micropolar model, non-Newtonian polymers, Deborah number, Keller-box method, heat transfer, boundary layers, skin friction, Nusselt number, thermal coating systems

K. Madhavi, Department of Mathematics, Madanapalle Institute of Technology and Science, Madanapalle-517325, India

*Corresponding Author: A. Subba Rao, Department of Mathematics, Madanapalle Institute of Technology and Science, Madanapalle517325, India

\section{Introduction}

Coating hydrodynamics has been an area of considerable interest since the monumental paper by Landau and Levich (1942) in which an elegant formulation was developed for the thickness of the film of a fluid which is deposited on a plate withdrawn vertically from a bath at constant velocity. This work was however confined to Newtonian viscous fluids. In many modern industries nonNewtonian fluids are encountered e.g. in polymer coating processes (Lawrence and Zhou, 1991). Numerous researchers have therefore investigated coating dynamics of different stationary or rotating geometrical bodies (plates, cones, spheres, cylinders) with non-Newtonian liquids and have employed a range of mathematical constitutive equations. Jenekhe and Schuldt (1984) studied coating flows of power-law and Carreau fluids on spinning disks. Campanella et al. (1986) investigated dip coating of a circular cylinder in non-Newtonian power-law fluids. Zevallos et al. (2005) presented a finite element simulation of forward roll coating flows of viscoelastic liquids using both Oldroyd-B and FENE-P models. These studies however ignored heat transfer which may be critical in certain coating systems (Mitsoulis, 1986). The diffusion of heat can modify polymer properties significantly (Mark, 1996). Several authors have therefore studied thermofluid transport in non-Newtonian external coating flows. Cao and Cui (2015) examined Ostwald-DeWaele pseudo-plastic fluids in free convection flow in porous media. Choudhury and

and Department of Mathematics, Sri Venkateswara University, Tirupati, India, E-mail: asrsvu@gmail.com

V. Ramachandra Prasad, Department of Mathematics, School of Advanced Sciences, VIT University, Vellore, India

0. Anwar Bég, Fluid Mechanics,Nanosystems and Propulsion, Aeronautical/Mechanical Engineering, School of Computing, Science, Engineering, Newton Bldg, University of Salford, Manchester M54WT, United Kingdom

A. Kadir, Materials, Corrosion and Structures, Petroleum and Gas Engineering, School of Computing, Science and Engineering, Newton Building, University of Salford, Manchester M54WT, United Kingdom 
Das (2016) employed the Reiner-Rivlin second order differential viscoelastic model for reactive magnetized thermosolutal convection in porous media. Thirumurugan and Vasanthakumari (2016) utilized the Walters B viscoelastic model to investigate the thermosolutal convection in twophase porous media transport. Prasad et al. (2013) and Subba Rao et al. (2016) investigated computationally the momentum and heat transfer characteristics in external boundary layer slip flow of a viscoplastic fluid from a cylinder.

The classical Navier-Stokes theory does not describe sufficiently the flow properties of polymeric fluids, colloidal suspensions, and fluids having certain additives. Eringen (1966) proposed therefore the theory of micropolar fluids which exhibit micro-rotation effects as well as micro-inertia. Micropolar fluids are fluids with microstructure. They belong to a class of fluids with non-symmetric stress tensor also known as polar fluids. Micropolar fluids include as a special case the conventional Navier-Stokes model of classical viscous fluids that we shall call ordinary fluids. Physically micropolar fluids consist of arbitrarily oriented particles suspended in a viscous medium. The formulation for micropolar fluid theory allows their implementation in boundary layer flows, of relevance to materials processing, and has resulted in considerable activity among researchers. Yacob et al., (2011) proposed a mathematical model to characterise the melting heat transfer in both stretching and shrinking sheet flows of a micropolar fluid. Prakash and Sinha (1976) applied micropolar fluid theory to simulate lubrication squeeze films for circular disks, deriving analytical expressions for film characteristics. Papautsky et al. (1999) employed micropolar fluid theory to study low speed flows in micro-machined rectangular metallic pipette arrays. Bhargava et al. (2016) analyzed the thermo-solutal convection in a cavity containing micro polar fluid with a variation finite element method. Latiff $e t$ al. (2015) used Maple software to analyse the multiple slip effects on transient forced bio convection flow of a micro polar nanofluid from a stretching/shrinking sheet with applications in materials fabrication. Alam et al. (2015) used Eringen's micropolar model to study transient FalknerSkan magnetic convection flows. Transport from external surfaces of curved bodies e.g. vertical cones has also stimulated some interest in recent years. Such flows are of relevance to chemical engineering systems and also materials synthesis. Cheng (2015) studied the free convection heat transfer from a non-isothermal permeable cone with suction and temperature-dependent viscosity. Solchana et al. (2016) analysed the momentum, heat and mass transfer behaviour of magneto hydrodynamic flow towards a vertical rotating cone in porous medium with thermophore- sis and Brownian motion effects. Cheng (2015) examined the natural convection heat transfer about a vertical cone embedded in a porous medium with isothermal wall conditions. Yih (1999) studied the effect of thermal radiation flux on free convection about a truncated cone. Gorla et al. (1986) investigated the micro polar convection boundary layer flow from a cone.

An interesting model in non-Newtonian fluid mechanics is the viscoelastic Jeffrey fluid model. This model degenerates to a Newtonian fluid at a very high wall shear stress. This fluid model also approximates reasonably well the rheological behaviour of a wide range of industrial liquids including biotechnological detergents, physiological suspensions, dense foams, geological sediments, cosmetic creams, syrups, etc. Many researchers have explored a range of industrial and biological flow problems using the Jeffery model. Prasad et al. (2014) and Subba Rao et al. (2015) studied external Jeffery viscoelastic boundary layer flow from a circular cylinder using an implicit finite difference code and showed that with increasing Deborah numbers there is a fall in Nusselt number (heat transfer rate) and the skin-friction coefficient. Tripathi and Bég (2013) analysed peristaltic propulsion with thermal diffusion using the Jeffery model. Hayat et al. (2015) simulated the polymeric thermal flow from a stretching cylinder in Jeffery viscoelastic liquid. Further studies of viscoelastic transport phenomena with the Jeffery's model include, Kodandapani et al. (2008), Nadeem et al. (2009), Hayat et al. (2012). The Jeffery model quite accurately describes the characteristics of relaxation and retardation times which arise in certain polymeric flows. Furthermore, this model utilizes time derivatives rather than converted derivatives, which facilitates numerical solutions in boundary value problems.

Relatively few studies have considered polymeric external boundary layer flows from curved bodies where the fluid possesses both microstructural and viscoelastic characteristics. Prasad et al. (2015) have studied nanoscale micropolar convection from a cylinder. However they did not consider viscoelastic effects. Thus, the objective of the current study is to examine the viscoelastic micropolar heat transfer in external boundary layer flow from a cone using the Jeffery non-Newtonian (2005) and Eringen micropolar models (2001). This is the novelty of this article.

\section{Mathematical Model}

The regime under investigation is natural convection boundary layer flows of incompressible viscoelastic mi- 


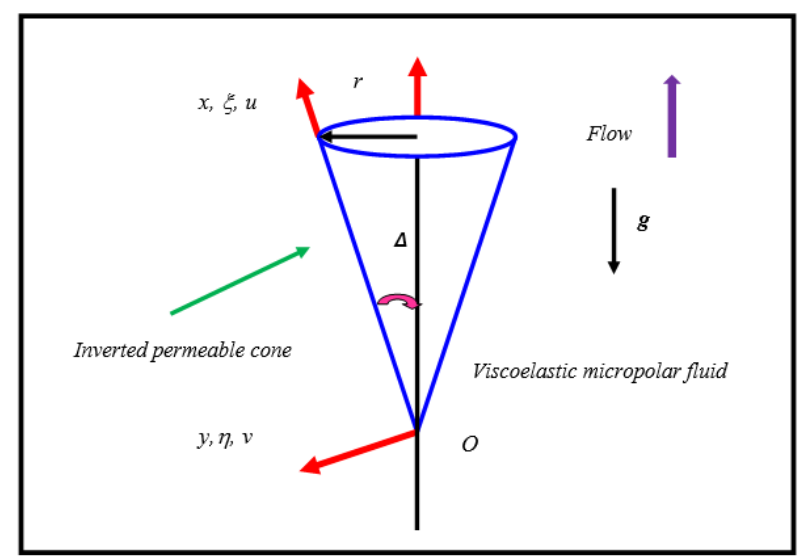

Fig. 1: Physical model and coordinate system

cropolar fluid (polymeric liquid) from a vertical isothermal solid cone, as shown in Fig. 1. Both cone and liquid are maintained initially at the same temperature. Fourier's law is assumed for heat conduction i.e. thermal relaxation effects are negated. Thermal stratification and dispersion are also neglected as is viscous heating. The flow is laminar, steady-state and the cone surface isothermal. These conditions correspond to steady polymeric convection in external boundary layer flow development on an axisymmetric cone geometry. Instantaneously the cone surface is raised to a temperature $T_{w}>T_{\infty}$, where the latter (ambient) temperature of the polymeric fluid is maintained as constant. The $x$-coordinate is measured along the slant surface of the cone from the origin (at the vertex) and the $y$ coordinate is directed normal to the cone surface. The cone has a semi-vertex angle of $A$.

With the Boussinesq buoyancy and boundary layer approximations, the governing equations for the conservation of mass, momentum, micro-rotation and energy can be written in two-dimensional Cartesian coordinates $(x, y)$ as follows:

$$
\frac{\partial(r u)}{\partial x}+\frac{\partial(r v)}{\partial y}=0
$$

$\rho\left(u \frac{\partial u}{\partial x}+v \frac{\partial u}{\partial y}\right)=(\mu+k) \frac{\partial^{2} u}{\partial y^{2}}+k \frac{\partial N}{\partial y}+\rho g \beta\left(T-T_{\infty}\right) \cos A$

$+\frac{v}{1+\lambda}\left[\frac{\partial^{2} u}{\partial y^{2}}+\lambda_{1}\left(u \frac{\partial^{3} u}{\partial x \partial y^{2}}-\frac{\partial u}{\partial x} \cdot \frac{\partial^{2} u}{\partial y^{2}}+\frac{\partial u}{\partial y} \cdot \frac{\partial^{2} u}{\partial x \partial y}\right.\right.$

$\left.+v \frac{\partial^{3} u}{\partial y^{3}}\right]$

$$
\begin{gathered}
u \frac{\partial T}{\partial x}+v \frac{\partial T}{\partial y}=\alpha \frac{\partial^{2} T}{\partial y^{2}} \\
\rho_{j}\left(u \frac{\partial N}{\partial x}+v \frac{\partial N}{\partial y}\right)=y^{\star} \frac{\partial^{2} N}{\partial y^{2}}-k\left(2 N+\frac{\partial u}{\partial y}\right)
\end{gathered}
$$

Here $r$ is the local radius of the truncated cone, and $v$ are the velocity components in $x$ and $y$ directions, Tand are the temperature and angular velocity of the viscoelastic micropolar fluid, $k$ and $j$ are the vortex viscosity and micro inertia density, $y^{\star}=(\mu+0.5 k) j$ is the spin gradient viscosity, $v=\frac{\mu}{\rho}$ is the kinematic viscosity of the viscoelastic micropolar fluid, $\mu$ is the dynamic viscosity of the viscoelastic micropolar fluid and $\rho$ is the fluid density. Furthermore, $\alpha$ is the thermal diffusivity, $\beta$ is the coefficient for thermal expansion and $g^{*}$ is the gravitational acceleration.With regard to the viscoelasticity in the polymeric liquid considered, this is simulated via the $\lambda$ parameter i.e. the ratio of relaxation to retardation times, and $\lambda_{1}$ parameter i.e. the retardation time. The following boundary conditions are imposed:

$$
\text { At } y=0, \quad u=v=0, \quad N=-\frac{1}{2} \frac{\partial u}{\partial y}, \quad T=T_{w}
$$

As $y \rightarrow \infty, \quad u \rightarrow 0, \quad v \rightarrow 0, \quad N \rightarrow 0, \quad T \rightarrow T_{\infty}$

Here $T_{\infty}$ is the free steam temperature. We introduce a stream function defined by the CauchyRiemannequations, $r u=\frac{\partial \psi}{\partial y}$ and $r v=-\frac{\partial \psi}{\partial x}$ and therefore, the mass conservation eqn. (1) is automatically satisfied. Furthermore, the following dimensionless variables are introduced into eqns. (2) - (4):

$$
\begin{aligned}
& \xi=\frac{\rho V_{w} X}{\mu}(G r)^{\frac{1}{4}}, \quad \eta=\frac{y}{\chi}(G r)^{\frac{1}{4}}, \\
& \psi=\frac{v r G r^{\frac{1}{4}}(f(\xi, \eta)+0.5 \xi)}{\rho}, \theta(\xi, \eta)=\frac{T-T_{\infty}}{T_{w}-T_{\infty}}, \\
& g=\frac{\rho x^{2} N}{\mu}(G r)^{\frac{-3}{4}}, \quad p r=\frac{\mu}{(\rho \alpha)} \\
& G r=\frac{\rho^{2} g^{\star} \beta\left(T_{w}-T_{\infty}\right) x^{3} \cos A}{v^{2}}, \quad D e=\frac{\lambda_{1} v(G r)^{\frac{1}{2}}}{x^{2}}
\end{aligned}
$$

Here all parameters are defined in the notation at the end of the article. The following non-dimensional partial differential equations for momentum and energy conservation therefore emerge:

$$
\begin{gathered}
{\left[\frac{4}{1+\lambda}+4(1+R)\right] f^{\prime \prime \prime}+7 f f^{\prime \prime}+4 \theta+4 \xi f^{\prime \prime}+4 R g^{\prime}-2\left(f^{\prime}\right)^{2}} \\
-\frac{D e}{1+\lambda}\left(2 f^{\prime} f^{\prime \prime \prime}-\left(f^{\prime \prime}\right)^{2}+7 f f^{i v}+4 \xi f^{i v}\right) \\
\frac{4}{\operatorname{Pr}} \theta^{\prime \prime}+7 f \theta^{\prime}+4 \xi \theta^{\prime}=\xi\left[f^{\prime} \frac{\partial \theta}{\partial \xi}-\theta^{\prime} \frac{\partial f}{\partial \xi}\right]
\end{gathered}
$$

$$
\begin{aligned}
& 4\left[1+\frac{R}{2}\right] g^{\prime \prime}-4 R B \xi^{2}\left(2 g+f^{\prime \prime}\right)+7 f g^{\prime}-f^{\prime} g+4 \xi g^{\prime} \\
& =\xi\left[f^{\prime} \frac{\partial g}{\partial \xi}-g^{\prime} \frac{\partial f}{\partial \xi}\right]
\end{aligned}
$$


The eight corresponding dimensionless boundary conditions emerge as:

$$
\begin{gathered}
\text { At } \eta=0, \quad f=0=f^{\prime}, \quad g=\frac{1}{2} \frac{\partial^{2} f}{\partial y^{2}}, \quad \theta=1 \\
\text { As } \eta \rightarrow \infty, \quad f^{\prime} \rightarrow 0, \quad f^{\prime \prime} \rightarrow 0, \quad g \rightarrow 0, \quad \theta \rightarrow 0
\end{gathered}
$$

Here primes denote the differentiation with respect to nondimensional transverse co-ordinate $\eta$ Here we also define the micropolar material parameters:

$$
B=v^{2}\left(j \rho^{2} v_{w}^{2}\right)^{-1} R=\frac{k}{v}
$$

The local Nusselt number of the inverted cone can be written as

$$
\frac{N u}{G r^{1 / 4}}=\theta^{\prime}(\xi, 0)
$$

In equation (12),

$$
N u=\frac{h x}{k}
$$

Here $h$ is the local heat transfer coefficient and $k$ is the thermal conductivity of the micropolar viscoelastic fluid.

\section{Numerical Solution With Keller Box Implict Method}

In this study the Keller-box implicit difference method is implemented to solve the non-linear eight order system of coupled boundary layer eqns. (7) - (9) subject to the boundary conditions (10). This method continues to be popular in boundary layer flows. Recent works employing Keller's box scheme include Cheng (2008) for micropolar non-isothermal flow from a truncated cone, Bég et al. (2016) for micro polar wall plume dynamics in permeable systems, Malik et al. (2015) and Subba Rao et al. (2017) for magnetic polymeric flows and Gaffar et al. (2015) and Subba Rao et al. $(2017,2018)$ for viscoelastic heat transfer from curved bodies. Very few of these papers however have provided guidance for researchers as to customization of the Keller-box scheme to heat transfer problems. We have included details of the discretization procedure below. Implementation of Keller's scheme involves the following four stages:

1. Reduction of the $N^{\text {th }}$ order partial differential equation system to $N$ first order equations

2. Finite Difference Discretization

3. Quasilinearization of Non-Linear Keller Algebraic Equations
4. Block-tridiagonal Elimination of Linear Keller Algebraic Equations

Step 1: Reduction of the $\boldsymbol{n}^{\text {th }}$ order partial differential equation system to $n$ first order equations

New variables are introduced to Eqns. (7) - (9) subject to the boundary conditions (10) are first written as a system of first order equations. For this purpose, we reset Eqns. (7) - (9) as a set of simultaneous equations by introducing the new variables:

$$
\begin{aligned}
& u(x, y)=f^{\prime}, \quad v(x, y)=f^{\prime \prime}, \quad q(x, y)=f^{\prime \prime \prime} \\
& s(x, y)=\theta, \quad t(x, y)=\theta^{\prime}, \quad p(x, y)=m^{\prime}
\end{aligned}
$$

$$
f^{\prime}=u
$$

$$
u^{\prime}=v
$$

$$
v^{\prime}=q
$$

$$
g^{\prime}=p
$$

$$
s^{\prime}=t
$$

$$
\begin{aligned}
& {\left[\frac{4}{1+\lambda}+4(1+R)\right] v^{\prime}+7 f v} \\
& +4 R P-2 u^{2}+4 s+4 \xi v-A\left[2 u q-v^{2}+7 f q^{\prime}+4 \xi q^{\prime}\right] \\
& =\xi\left[\left(u \frac{\partial u}{\partial \xi}-v \frac{\partial f}{\partial \xi}\right)-A\left(u \frac{\partial q}{\partial \xi}-q \frac{\partial u}{\partial \xi}+v \frac{\partial v}{\partial \xi}-q^{\prime} \frac{\partial f}{\partial \xi}\right)\right]
\end{aligned}
$$

$$
\begin{gathered}
\frac{4}{\operatorname{Pr}} t^{\prime}+7 f t+4 \xi t=\xi\left(u \frac{\partial s}{\partial \xi}-t \frac{\partial f}{\partial \xi}\right) \\
4\left(1+\frac{R}{2}\right) p^{\prime}-4 R B \xi^{2}(2 m+v)+7 f p-u m+4 \xi p \\
=\xi\left(u \frac{\partial m}{\partial \xi}-p \frac{\partial f}{\partial \xi}\right)
\end{gathered}
$$

The associated boundary conditions are

$$
\begin{array}{r}
\text { At } \eta=0, \quad f=0, u=0, \quad s=1, \quad g=0 \\
\text { As } \eta \rightarrow \infty, u \rightarrow 0, v \rightarrow 0, s \rightarrow 0, g \rightarrow 0
\end{array}
$$

\section{Phase b: Finite Difference Discretization}


A two dimensional computational grid is imposed on the $\xi-\eta$ plane as sketched in Fig. 1. The stepping process is defined by:

$$
\begin{gathered}
\eta_{0}=0, \eta_{j}=\eta_{j-1}+h_{j}, j=1,2, \ldots, J, \\
\xi^{0}=0, \xi^{n}=\xi^{n-1}+k_{n}, n=1,2 .
\end{gathered}
$$

where $k_{n}$ and $h_{j}$ denote the step distances in the $\xi$ and $\eta$ directions respectively.

If $g_{j}^{n}$ denotes the value of any variable at $\left(\eta_{j}, \xi^{n}\right)$, then the variables and derivatives of Equations. (16) - (23) at $\left(\eta_{j-1 / 2}, \xi^{n-1 / 2}\right)$ are replaced by:

$$
\begin{gathered}
g_{j-1 / 2}^{n-1 / 2}=\frac{1}{4}\left(g_{j}^{n}+g_{j-1}^{n}+g_{j}^{n-1}+g_{j-1}^{n-1}\right), \\
\left(\frac{\partial g}{\partial \eta}\right)_{j-1 / 2}^{n-1 / 2}=\frac{1}{2 h_{j}}\left(g_{j}^{n}-g_{j-1}^{n}+g_{j}^{n-1}-g_{j-1}^{n-1}\right), \\
\left(\frac{\partial g}{\partial \xi}\right)_{j-1 / 2}^{n-1 / 2}=\frac{1}{2 k^{n}}\left(g_{j}^{n}-g_{j-1}^{n}+g_{j}^{n-1}-g_{j-1}^{n-1}\right),
\end{gathered}
$$

We now state the finite-difference approximation of equations. (16) - (23) for the $\left(\eta_{j-1 / 2}, \xi^{n}\right)$, below

$$
\begin{aligned}
& h_{j}^{-1}\left(f_{j}^{n}-f_{j-1}^{n}\right)=u_{j-1 / 2}^{n}, \\
& h_{j}^{-1}\left(u_{j}^{n}-u_{j-1}^{n}\right)=v_{j-1 / 2}^{n}, \\
& h_{j}^{-1}\left(v_{j}^{n}-v_{j-1}^{n}\right)=q_{j-\frac{1}{2}}^{n} \\
& h_{j}^{-1}\left(g_{j}^{n}-g_{j-1}^{n}\right)=p_{j-\frac{1}{2}}^{n} \\
& h_{j}^{-1}\left(\theta_{j}^{n}-\theta_{j-1}^{n}\right)=t_{j-\frac{1}{2}}^{n} \\
& \left.+\frac{4}{1+\lambda}+4(1+R)\right]\left(\frac{v_{j}-v_{j-1}}{h_{j}}\right) \\
& +(\alpha+7) \frac{1}{4}\left(f_{j}+f_{j-1}\right)\left(v_{j}+v_{j-1}\right) \\
& +4 R \frac{1}{2}\left(p_{j}+p_{j-1}\right)-(\alpha+2) \frac{1}{4}\left(u_{j}+u_{j-1}\right)^{2}+4 \frac{1}{2}\left(s_{j}+s_{j-1}\right) \\
& +4 \xi \frac{1}{2}\left(v_{j}+v_{j-1}\right)-2 A \frac{1}{4}\left(u_{j}+u_{j-1}\right)\left(q_{j}-q_{j-1}\right) \\
& +A(1+\alpha) \frac{1}{4}\left(v_{j}+v_{j-1}\right)^{2}-A(7+\alpha) \frac{1}{2}\left(f_{j}+f_{j-1}\right)\left(\frac{q_{j}-q_{j-1}}{h_{j}}\right) \\
& -4 A \xi\left(\frac{q_{j}-q_{j-1}}{h_{j}}\right)-\frac{\alpha}{2}\left(v_{j}+v_{j-1}\right) f^{n-1}+\frac{\alpha}{2}\left(f_{j}+f_{j-1}\right) v^{n-1} \\
& +2 A \frac{\alpha}{2}\left(u_{j}+u_{j-1}\right) q^{n-1}+2 A \frac{\alpha}{2}\left(q_{j}+q_{j-1}\right) u^{n-1} \\
& +A \alpha\left(\frac{q_{j}-q_{j-1}}{h_{j}}\right) f^{n-1}-A \frac{\alpha}{2}\left(f_{j}+f_{j-1}\right) q^{\prime n-1}=-\left[R_{1}\right]^{n-1}
\end{aligned}
$$

$$
\begin{aligned}
& \frac{4}{p_{r}}\left(t_{j}-t_{j-1}\right)+\frac{h_{j}}{4}(7+\alpha)\left(f_{j}+f_{j-1}\right)\left(t_{j}+t_{j-1}\right) \\
& +2 h_{j} \xi\left(t_{j}+t_{j-1}\right)-\frac{h_{j} \alpha}{4}\left(u_{j}+u_{j-1}\right)\left(s_{j}+s_{j-1}\right) \\
& +\frac{h_{j} \alpha}{2}\left(u_{j}+u_{j-1}\right) s^{n-1}-\frac{h_{j} \alpha}{2}\left(s_{j}+s_{j-1}\right) u^{n-1} \\
& -\frac{h_{j} \alpha}{2}\left(t_{j}+t_{j-1}\right) f^{n-1}+\frac{h_{j}}{2} \alpha\left(f_{j}+f_{j-1}\right) t^{n-1}=-\left[R_{2}\right]^{n-1}
\end{aligned}
$$

$$
\begin{aligned}
& 4\left(1+\frac{R}{2}\right)\left(p_{j}-p_{j-1}\right)-\frac{8 R B \xi^{2} h_{j}}{2}\left(m_{j}+m_{j-1}\right) \\
& -\frac{4 R B \xi^{2} h_{j}}{2}\left(v_{j}+v_{j-1}\right)+\frac{(\alpha+7) h_{j}}{4}\left(f_{j}+f_{j-1}\right)\left(p_{j}+p_{j-1}\right) \\
& -\frac{(\alpha+1) h_{j}}{4}\left(u_{j}+u_{j-1}\right)\left(m_{j}+m_{j-1}\right)+\frac{4 \xi h_{j}}{2}\left(p_{j}+p_{j-1}\right) \\
& +\frac{\alpha h_{j}}{2}\left(u_{j}+u_{j-1}\right) m^{n-1}-\frac{\alpha}{2}\left(m_{j}+m_{j-1}\right) u^{n-1} \\
& -\frac{\alpha h_{j}}{2}\left(p_{j}+p_{j-1}\right) p^{n-1}=-\left[R_{3}\right]^{n-1}
\end{aligned}
$$

where we have used the abbreviations

$$
\alpha=\frac{\xi^{n-1 / 2}}{k_{n}}
$$

$$
\begin{gathered}
{\left[R_{1}\right]_{j-\frac{1}{2}}^{n-1}=} \\
h_{j}\left[\begin{array}{c}
\frac{4}{1+\lambda}+4(1+R) v^{\prime}-(\alpha-7) f v+4 R P+(\alpha-2) u^{2}+4 s \\
+4 \xi v-2 A(u q)-A(\alpha-1) v^{2}+A(\alpha-7) f q^{\prime}-4 A \xi q^{\prime}
\end{array}\right] \\
{\left[R_{2}\right]_{j-\frac{1}{2}}^{n-1}=h_{j}\left[\frac{4}{p_{r}} t^{\prime}-(\alpha-7) f t+\alpha(u s)+4 \xi t\right]} \\
{\left[R_{3}\right]_{j-\frac{1}{2}}^{n-1}=h_{j}\left[\begin{array}{c}
4\left(1+\frac{R}{2}\right) p^{\prime}-8 R B \xi^{2} m-4 R B \xi^{2} v \\
-(\alpha-7)(f p)+(\alpha-1)(u m)+4 \xi p
\end{array}\right]}
\end{gathered}
$$

The boundary conditions are

$f_{0}^{n}=u_{0}^{n}=0, s_{0}^{n}=1, m_{0}^{n}=1, u_{j}^{n}=0, v_{j}^{n}=0, s_{j}^{n}=0, m_{j}^{n}=0$

Phase c: Quasilinearization of Non-Linear Keller Algebraic Equations

If we assume $f_{j}^{n-1}, u_{j}^{n-1}, v_{j}^{n-1}, s_{j}^{n-1}, t_{j}^{n-1}, q_{j}^{n-1}$ $m_{j}^{n-1}, p_{j}^{n-1}$ to be known for the solution of $8 \mathrm{~J}+8$ equations for the solution of $8 \mathrm{~J}+8$ unknowns $f_{j}^{n}, u_{j}^{n}, v_{j}^{n}, s_{j}^{n}, t_{j}^{n}, q_{j}^{n}, m_{j}^{n}, p_{j}^{n}, j=0,1,2 \ldots J$. This non - linear system of algebraic equations is linearized by means of Newton's method and this method was used by Subba Rao et al. (2017).

Phase d: Block-tridiagonal Elimination of Linear Keller Algebraic Equations 
The linear system is solved using the blockelimination method, since it possess a block-tridiagonal structure consists of variables or constants. The system consists of block matrices the complete linearized system is formulated as a block matrix system, where each element in the co-efficient matrix is a matrix itself. Then, this system is solved using the efficient Keller-box method. The numerical results are strongly influenced by the number of mesh points in both directions. After some trials in the $\eta$-directions (transverse i.e. radial coordinate) a larger number of mesh points are selected whereas in the $\xi$-directions (tangential coordinate) significantly less mesh points are necessary. The numerical algorithm is executed in MATLAB on a PC. The method demonstrates excellent stability, convergence and consistency (Keller, 1978).

\section{Keller Box Method (KBM) Numerical Results and Discussion}

Table 1: Numerical values of skin-friction and heat transfer coefficient for different values of $\xi$ while $P r=0.71, S_{f}=0.5$ and $S_{T}=1.0$ when $\beta \rightarrow \infty$ (Newtonian case).

\begin{tabular}{clccc}
\hline \multirow{2}{*}{$\xi$} & \multicolumn{2}{c}{ Alam et.al results [19] } & \multicolumn{2}{c}{ Present results } \\
\cline { 2 - 5 } & $f^{\prime \prime}(\xi, 0)$ & $-\theta^{\prime}(\xi, 0)$ & $f^{\prime \prime}(\xi, 0)$ & $-\theta^{\prime}(\xi, 0)$ \\
\hline 0.0 & 0.891936 & 0.420508 & 0.892157 & 0.421782 \\
0.5 & 0.896407 & 0.620748 & 0.897843 & 0.619852 \\
1.0 & 0.856963 & 0.848082 & 0.848784 & 0.840231 \\
1.5 & 0.779210 & 1.129365 & 0.771562 & 1.119786 \\
2.0 & 0.674111 & 1.441742 & 0.675320 & 1.432852 \\
3.0 & 0.434152 & 2.202662 & 0.412372 & 2.063725 \\
\hline
\end{tabular}

Moreover, in order to verify the accuracy of present method, the analytical results obtained in the present work were compared with those available in the literature, obtaining an excellent agreement with those given in Alam et al. [19] for particular values of $\xi$. The table presents the influence of increasing the tangential coordinate $\xi$ on the skinfriction and local nussult number results. Increaseing $\xi$, is found to decrease skinfriction and increse heat transfer rate. There is a vast change in skin friction and heat transfer rate when taking the larger values of $\xi$.

Comprehensive solutions have been obtained and are presented in Figs. 2 - 19. We examine the influence of several key parameters, namely Deborah number $(D e)$, ratio of relaxation to retardation times $(\lambda)$, Prandtl number $(P r)$, micropolar parameter $(R)$ i.e. vortex to dynamic viscosity

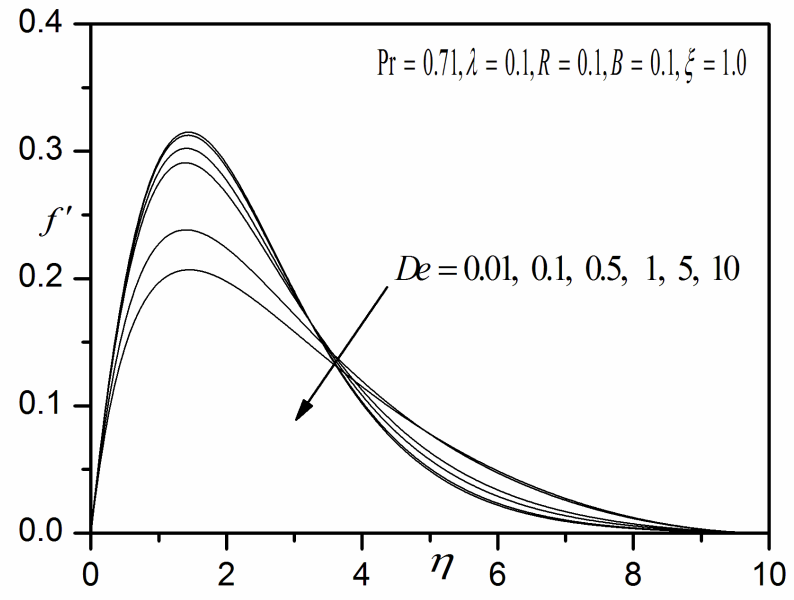

Fig. 2: Influence of De on velocity.

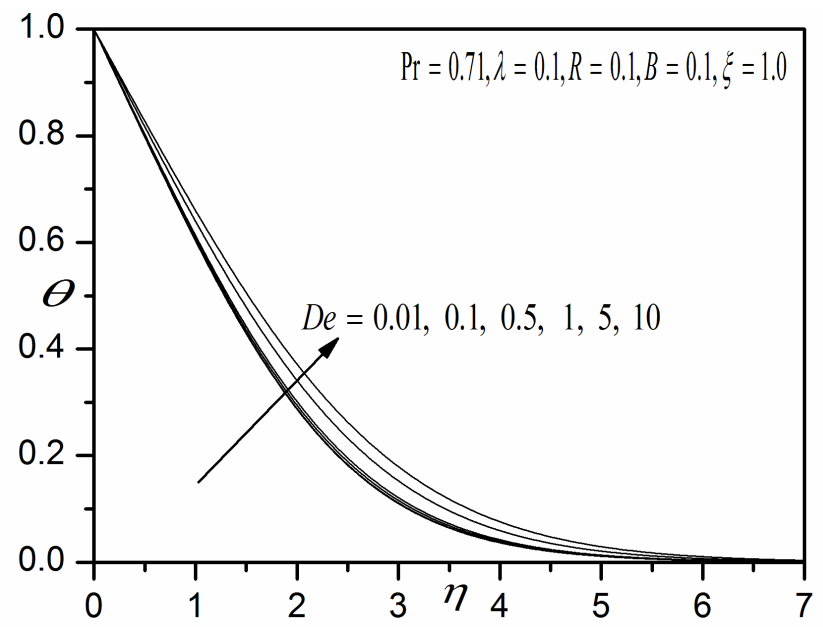

Fig. 3: Influence of $D e$ on the temperature.

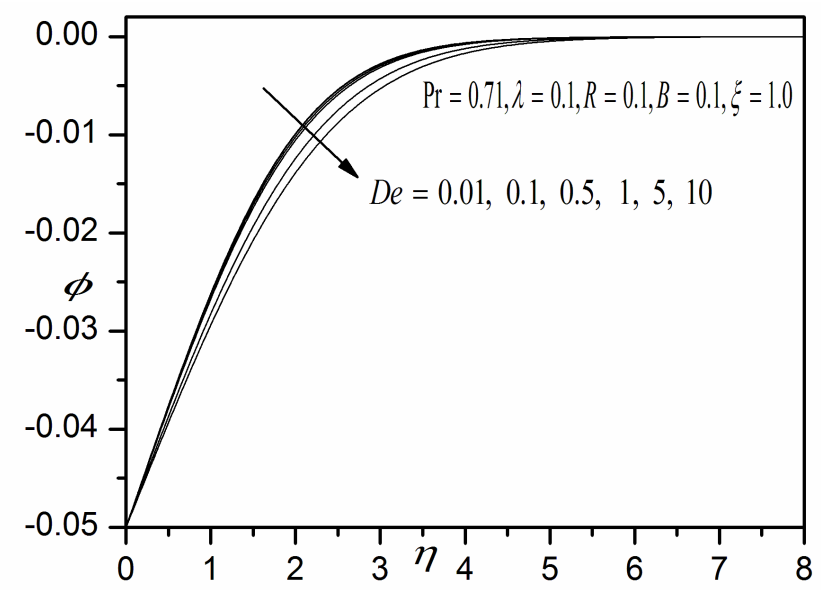

Fig. 4: Influence of $D e$ on the angular velocity. 


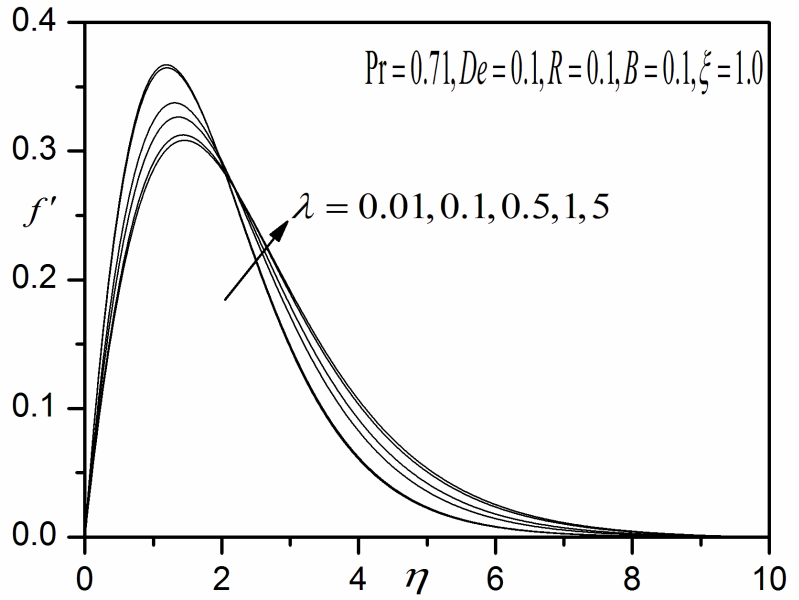

Fig. 5: Influence of $\lambda$ on the velocity.

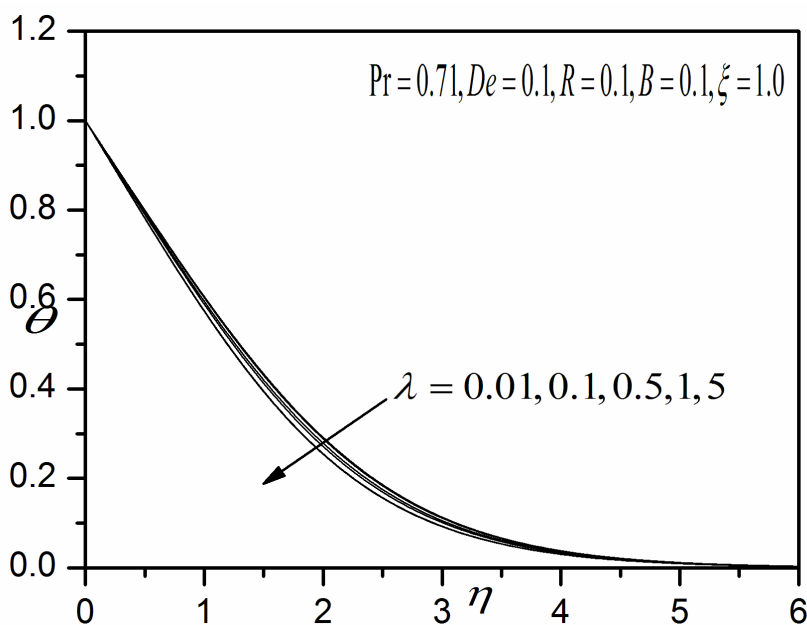

Fig. 6: Influence of $\lambda$ on the temperature.

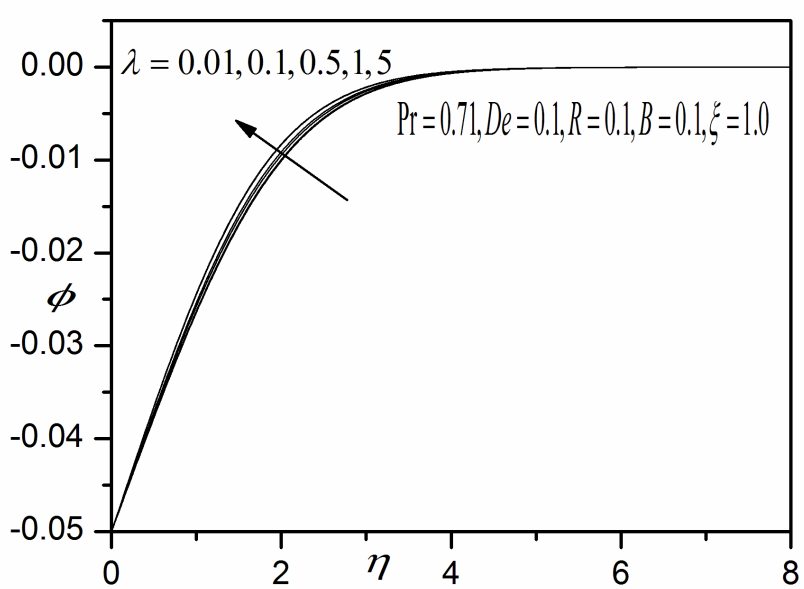

Fig. 7: Influence of $\lambda$ on the angular velocity.

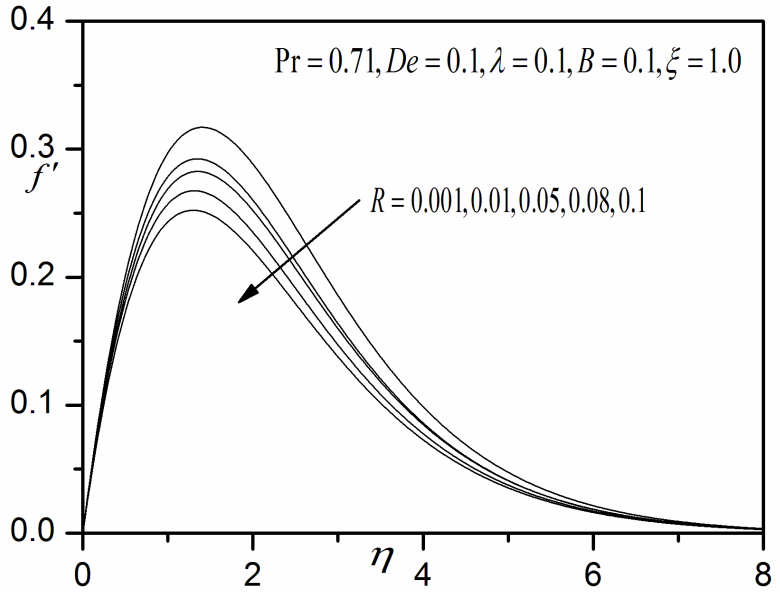

Fig. 8: Influence of $R$ on the velocity.

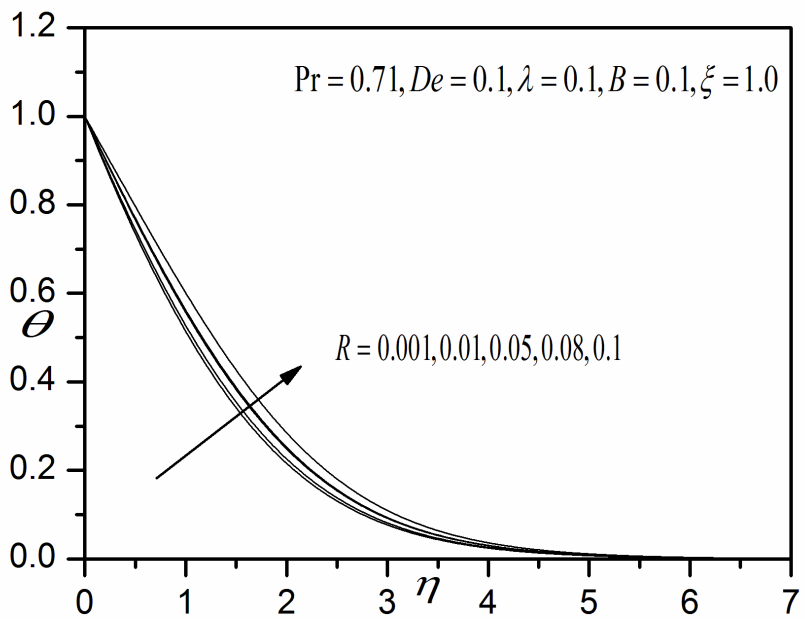

Fig. 9: Influence of $R$ on the temperature.

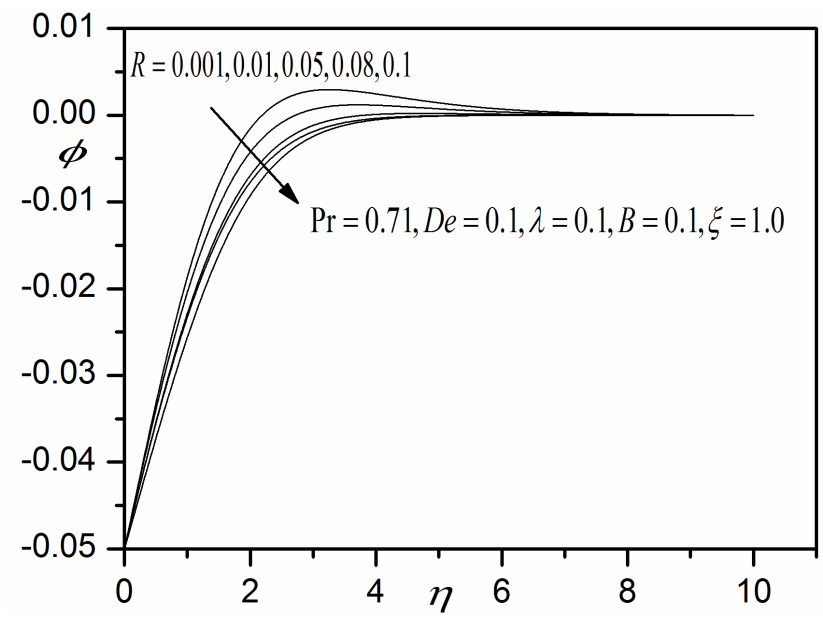

Fig. 10: Influence of $R$ on the angular velocity. 


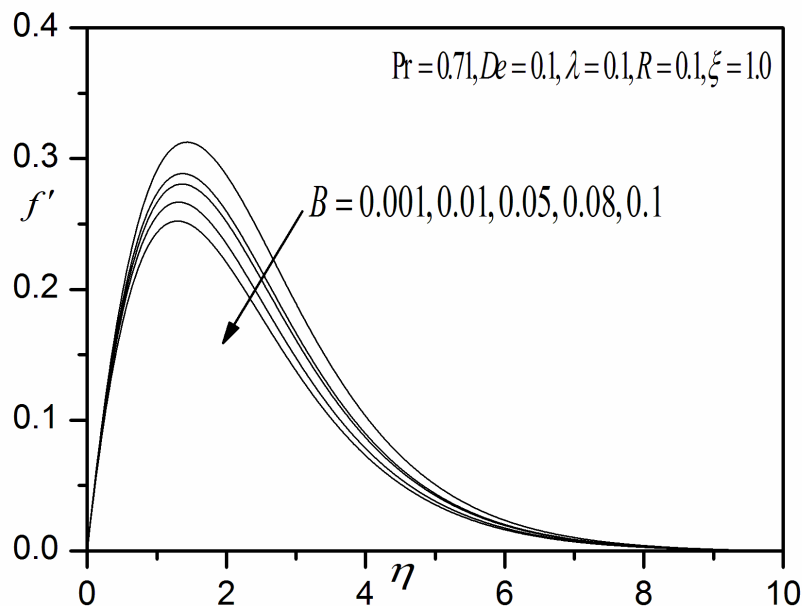

Fig. 11: Influence of $B$ on the velocity.

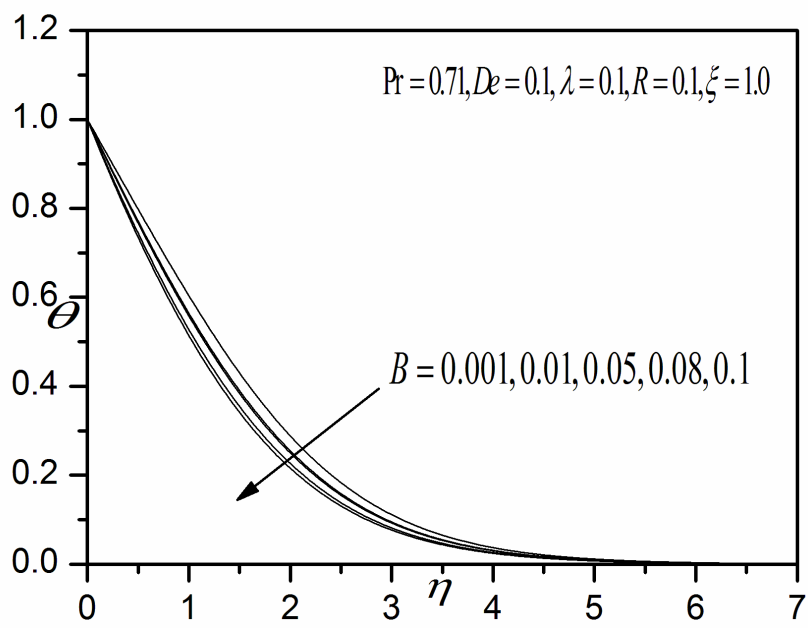

Fig. 12: Influence of $B$ on the temperature.

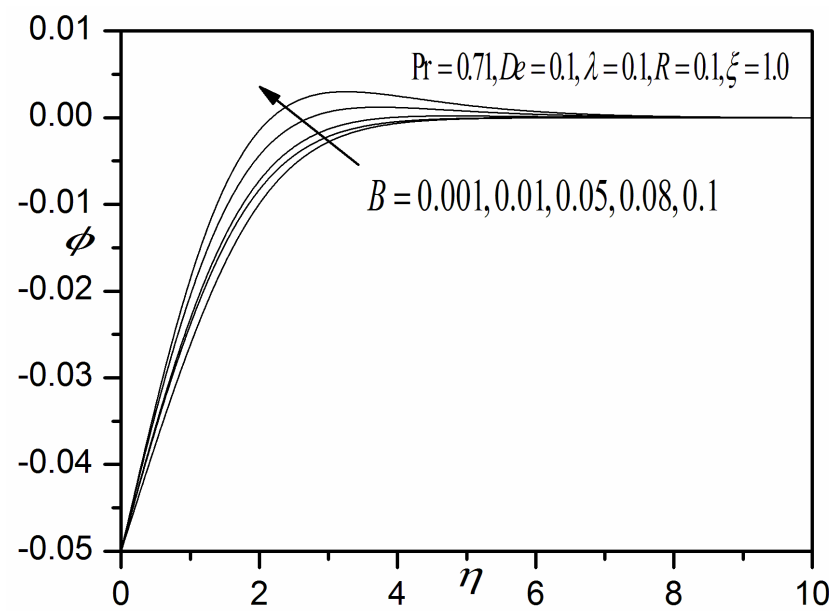

Fig. 13: Influence of $B$ on the angular velocity.

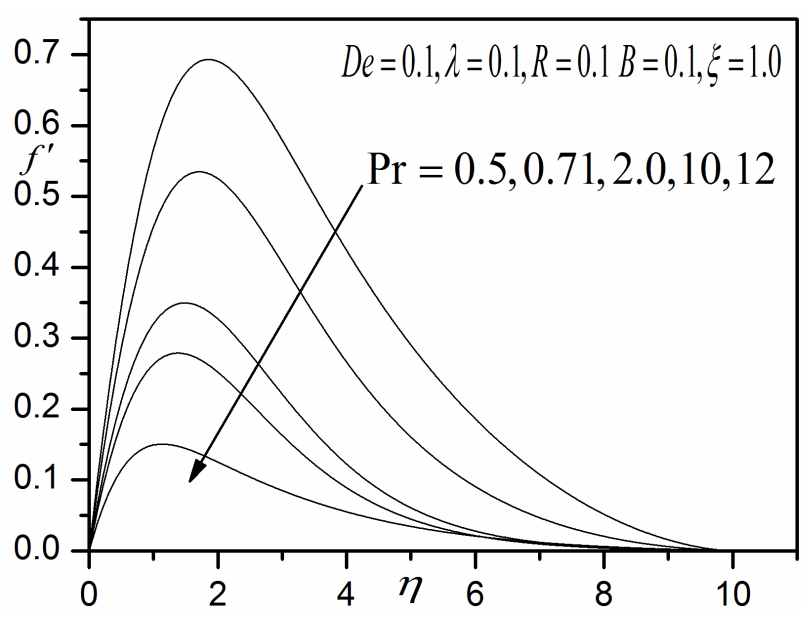

Fig. 14: Influence of $\operatorname{Pr}$ on the velocity.

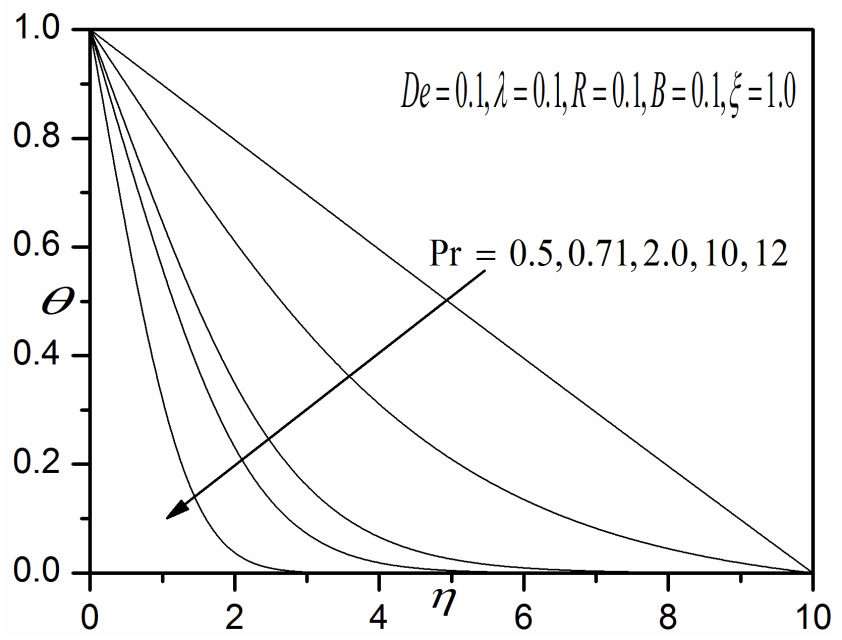

Fig. 15: Influence of $\operatorname{Pr}$ on the temperature.

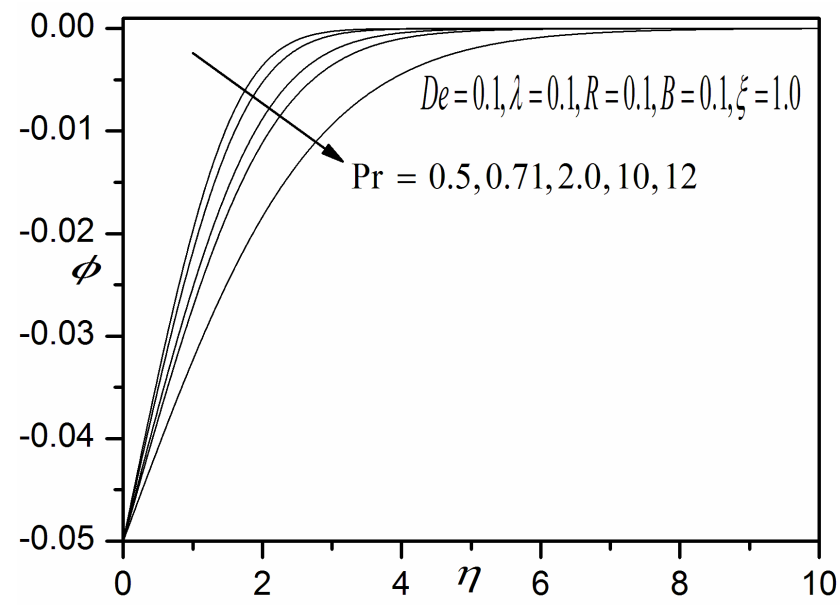

Fig. 16: Influence of $\operatorname{Pr}$ on the angular velocity. 


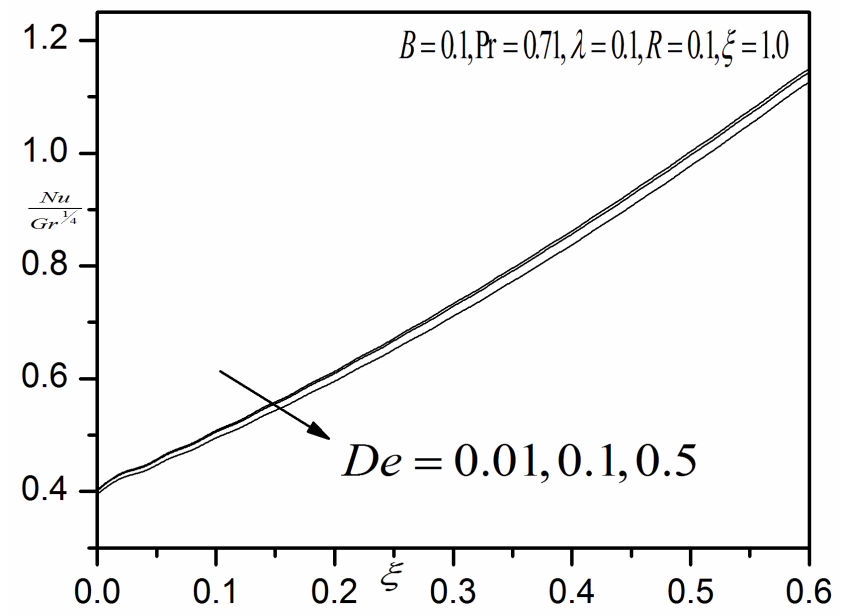

Fig. 17: Influence of $D e$ on Nusselt number.

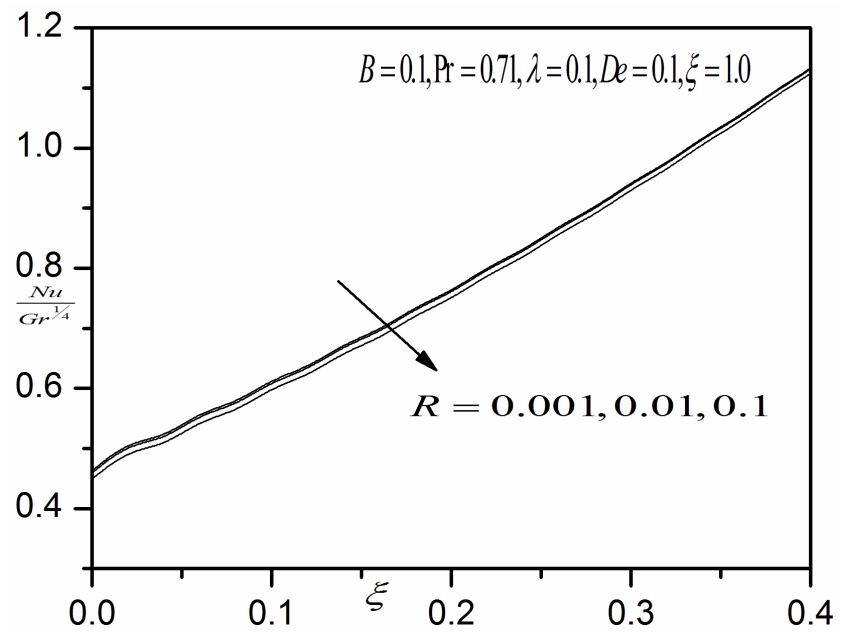

Fig. 18: Influence of $R$ on the Nusselt number.

ratio and micro-inertia density parameter $(B)$. The effect of the stream wise coordinate $(\xi)$ is not explicitly examined. Default parameter values prescribed are: $D e=0.1, \lambda=0.1$, $\operatorname{Pr}=0.71, R=0.1, B=0.1, \xi=1.0$. In the graphs the angular velocity $(g)$ is denoted as $\varphi$.

Figures $2-4$, illustrate the influence of Deborah number on velocity $\left(f^{\prime}\right)$, temperature $(\theta)$ and angular velocity $(\phi)$. Dimensionless velocity component (Fig. 2) is considerably reduced with increasing $D e$ values. De arises in connection with some higher order derivatives in the momentum boundary layer equations, (7), i.e., $-\frac{D e}{1+\lambda}\left(2 f^{\prime} f^{\prime \prime \prime}-\left(f^{\prime \prime}\right)^{2}+7 f f^{i v}+4 \xi f^{i v}\right)$ and also $\xi\left(-\frac{D e}{1+\lambda}\left(f^{\prime} \frac{\partial f^{\prime \prime \prime}}{\partial \xi}-f^{\prime \prime \prime} \frac{\partial f^{\prime}}{\partial \xi}+f^{\prime \prime} \frac{\partial f^{\prime \prime}}{\partial \xi}-f^{i v} \frac{\partial f}{\partial \xi}\right)\right)$. This parameter therefore exerts a significant influence on shearing characteristics of the polymer flow. Deborah number is defined as the ratio of the characteristic time to the time scale of deformation. For a fixed value of the characteristic

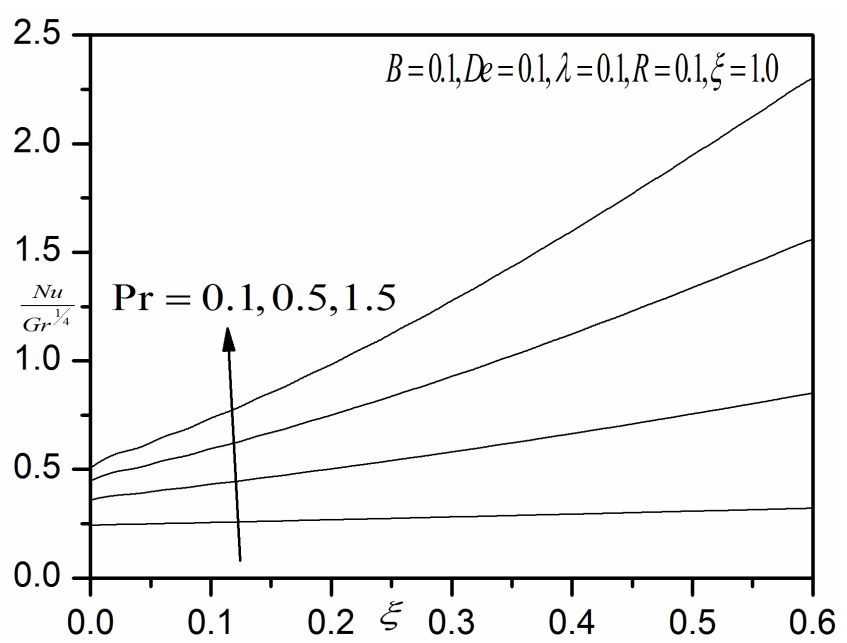

Fig. 19: Influence of $\operatorname{Pr}$ on the Nusselt number result.

time, there may be different values of the time scale of deformation and hence there can be many different Deborah number values for the same polymer. If $D e>1.0$, elastic effects are dominant while if $D e<0.5$, viscous effects prevail. For any values other than these two extremes, the material would depict viscoelastic behaviour. For polymers i.e., for non-Newtonian fluids, higher $D e$ values correspond to the polymer becoming highly oriented in one direction and stretched. For very high $D e$ values, the fluid movement is too fast for elastic forced to relax and the material acts as a purely elastic fluid. For small De values, the time scale of fluid moment is much greater than the relaxation time of elastic forces in the polymer. In Fig. 3 an increase in Debroah number enhance temperature throughout the boundary layer regime. This has also been observed by Hayat et al. (2012). In Fig. 4 with increasing De values, there is a substantial decrease in angular velocity i.e. microelement rotary motion is inhibited. The heat transfer rate is substantially decreased with increasing $D e$ values. There is a progressive decay in heat transfer rate with incresing the tangential coordinate. A decrease in heat transfer rate at the wall imply less heat is convected from the fluid regime to the cone, thereby heating the boundary layer.

Figures 5-7 present the effect of the ratio of relaxation to retardation times i.e., $\lambda$ on velocity $\left(f^{\prime}\right)$, temperature $(\theta)$ and angular velocity $(\phi)$ distributions throughout the boundary layer regime. A significant increase in velocity is observed with increasing $\lambda$ values. Conversely, temperature is markedly reduced with increasing values of $\lambda$. Also increasing $\lambda$ is seen to increase the angular velocity significantly i.e. encourage spin of the micro-elements. 
Velocity is significantly incresed with increasing $\lambda$. The polymer flow is therefore considerbly accelarated with an increase in relaxation time (or decrease in retardation time).

The mathematical model reduces to the Newtonian viscous flow model as $\lambda \rightarrow$ Oand $D e \rightarrow 0$, since this negates relaxation, retadation and elasticity effects.The momentum boundary layer equation in theese cases contracts to the familiar equation for Newtonian mixed convection from the cone:

$$
\begin{aligned}
& {[4+4(1+R)] f^{\prime \prime \prime}+7 f^{\prime \prime}+4 \theta+4 \xi f^{\prime \prime}+4 R g^{\prime}-2\left(f^{\prime}\right)^{2}} \\
& =\xi\left[f^{\prime} \frac{\partial f^{\prime}}{\partial \xi}-f^{\prime \prime} \frac{\partial f}{\partial \xi}\right]
\end{aligned}
$$

Effectively with greater relaxation time of the polymer the thermal boundary layer thickness is reduced.

Figures $8-10$ depicts the effect of the vortex viscosity parameter $(R)$ on velocity $\left(f^{\prime}\right)$, temperature $(\theta)$ and angular velocity $(\phi)$. For $R=1$, the micropolar and Newtonian dynamic viscosity are equivalent. For $R=0$, micropolarity is neglected and the equations reduce to the nonpolar case. Although relatively simple in definition, $R$, has a prominent influence on all the flow variables. We observe in Fig. 8 that an increase in $R$ strongly decelerates the flow i.e. damps linear velocity. Fig. 9 demonstrates that with stronger micro-polarity i.e. greater $R$ value, the temperatures are elevated in the boundary layer. The regime is significantly heated and thermal boundary layer thickness is increased. The increased vortex viscosity encourages thermal diffusion i.e. energizes the flow. This increases the efficiency of thermal convection within the body of the fluid from the microscopic to the macroscopic scale and effectively transports heat with greater intensity from the cone surface into the fluid regime. Fig. 11 depicts that strong reversal of micro-element rotation is induced very close to the wall (cone surface) with increasing $R$ values.

Figures $11-13$ depict the influence of velocity $\left(f^{\prime}\right)$, temperature $(\theta)$ and angular velocity for different values of material parameter $B$. It is observed that an increase of $B$ significantly decelerates the flow, i.e. depresses velocity throughout the boundary layer regime. The temperature is also decreased with increasing $B$ values. Conversely with increasing material parameter, $B$, the angular velocity (micro-rotation) is increased, indicating that faster gyratory motions of micro-elements are induced.

Figures $14-16$ represent typical profiles for $\left(f^{\prime}\right)$, temperature $(\theta)$ and angular velocity $(\phi)$ for various values of $P r$. It is observed that an increase in $P r$ decreases in velocity. The most prominent variation in profiles arises at intermediate distances from the cone surface. Furthermore, increasing $\operatorname{Pr}$ generates a substantial reduction in the fluid temperature and the thermal boundary layer thickness. At large $P r$, the thermal boundary layer is thinner than at a smaller Pr. This is associated with the fact that for small Prandtl numbers the fluid has very high thermal conductivity. An increase in $P r$ from 0.5 through $0.71,2.0,10,12$ as shown in Fig. 14, substantially decelerates the flow i.e. reduces hydrodynamic boundary layer thickness. Similarly higher Prandtl number, which corresponds to lower thermal conductivity of the polymer results in a marked depression in temperatures (fig. 16). The lower thermal conductivity of denser polymers inhibits thermal diffusion via conduction heat transfer. Less heat is therefore conveyed from the cone surface (wall) into the boundary layer and thermal boundary layer thickness is decreased.

Increasing $P r$ as observed in Fig. 16, reduces angular velocity i.e stifles micro-element spin (angular deceleration).

Figures $17-19$ presents the Nusselt Number comparisons for different values of $D e, R$ and $P r$ respectively: Increasing Deborah number, decrease the Nusselt number i.e reduces heat transfer from the viscoelastic micropolar fluid to the cone wall. Increasing vortex viscosity parameter, $R$ is also seen to decrease the Nusselt number. Increasing Prandtl number, $P r$ is observed to enhance the Nusselt number since temperatures are reduced in the fluid and greater heat is convected to the cone surface. This inevitably has implications for cooling of thin film coatings.

\section{Conclusions}

A mathematical model has been developed for the buoyancy-driven, natural convection boundary layer flow of a viscoelastic micropolar fluid from a vertical isothermal cone. The Jeffery non-Newtonian and Eringen microstructural models have been used to simulate different rheological characteristics. The transformed boundary layer conservation equations have been solved with prescribed boundary conditions using the finite difference implicit Keller-box method which has second order accuracy. A comprehensive assessment of the effects of Deborah number $D e$, ratio of retardation to relaxation time $\lambda$, vortex viscosity parameter, Prandtl number $\mathrm{Pr}$, micro-inertia density parameter $B$. Excellent convergence and stability characteristics are demonstrated by the Keller box scheme which is capable of solving very strongly nonlinear rheological problems.

The present simulations have shown that:

(i) Increasing Deborah number, De reduces velocity and Nusselt number whereas it increases temperature and an- 
gular velocity (micro-rotation of micro-elements).

(ii) Increasing ratio of retardation to relaxation time, $\lambda$ increases the velocity and angular velocity whereas it reduces the temperature (and thermal boundary layer thickness).

(iii) Increasing Eringen vortex viscosity parameter, $R$ decreases the velocity and angular velocity whereas it elevates temperature.

(iv) Increasing micro-inertia density parameter, $B$, reduces velocity and temperature whereas it enhances angular velocity.

(v) Increasing Prandtl number, $P r$ decreases velocity, temperature and angular velocity.

(vi) Increasing Deborah number and vortex viscosity parameter both depress Nusselt number whereas an increase in Prandtl number enhances the Nusselt number (heat transfer rate at the cone surface).

The current study has neglected rotation of the cone and therefore Coriolis body force effects, which are also relevant in polymer chemical processing operations, for example in spin coating processes. These will be considered in the near future.

Acknowledgement: This work is financially supported by the DST with reference no.SR/WOS-A/MS-09/2014(G). The authors appreciate the constructive comments of the reviewers which led to definite improvement in the paper. The authors are thankful to the management of Madanapalle Institute of Technology \& Science, Madanapalle for providing research facilities in the campus.

\section{References}

[1] Landau L.D. and Levich B., Dragging of liquid by a plate, Acta Physiochim. USSR, 1942, 17, 42-54

[2] Lawrence C.J., Zhou W., Spin coating of non-Newtonian fluids, J. Non-Newtonian Fluid Mechanics, 1991, 39, 137-187

[3] Samson Jenekhe A., Spencer B. Schuldt, Coating flow of nonNewtonian fluids on a flat rotating disk, Ind. Eng. Chem. Fundamen., 1984, 23(4), 432-436

[4] Osvaldo Campanella H., Jorge L. Galazzo, Ramón L. Cerro, Viscous flow on the outside of a horizontal rotating cylinder-II. Dip coating with a non-Newtonian fluid, Chemical Engineering Science, 1986, 41(11), 2707-2713

[5] Zevallos G.A., Carvalhoa M.S., Pasquali M. Forward roll coating flows of viscoelastic liquids, J. Non-Newtonian Fluid Mech., 130, 96-109 (2005).

[6] Mitsoulis E., Fluid flow and heat transfer in wire coating: A review, Advances in Polymer Technology, 1986, 6, 467-487

[7] Mark J.E., Physical Properties of Polymers Handbook, AIP Press, Woodbury, New York, USA, 1996
[8] Yonghua Cao and Xiaomin Cui, Natural convection of power law fluids in porous media with variable thermal and mass diffusivity, International Journal of Heat and Technology, 2015, 33(2), 85-90.

[9] Rita Choudhury and Bandita Das, Influence of visco-elasticity on MHD heat and mass transfer flow through a porous medium bounded by an inclined surface with chemical reaction, International Journal of Heat and Technology, 2016, 34(2), 332-338

[10] K. Thirumurugan and R. Vasanthakumari, Double - diffusive convection of non - Newtonian Walters' (MODELB') viscoelastic fluid through brinkman porous medium with suspended particles, International Journal of Heat and Technology, 2016, 34(3), 357-363

[11] Prasad V. R., Subba Rao A., Bhaskar Reddy N., Vasu B and Anwar Beg O., Modelling laminar transport phenomena in a Casson rheological fluid from a horizontal circular cylinder with partial slip, Proc. IMECHE Part E : J. Process Mechanical Engineering, 2013, 227(4), 309-326

[12] Subba Rao. A, V.R.Prasad, K. Harshavalli and O. Anwar Bég, Thermal radiation effects on non- Newtonian fluid in a variable porosity regime with partial slip, J. Porous Media, 2016, 19(4), 313-329

[13] Eringen A.C., Theory of micropolar fluids. J. Math. Mech., 1966, 16, 1-16

[14] Yacob N. A., Ishak A., Pop I., Melting heat transfer in boundary layer stagnation-point flow towards a stretching/shrinking sheet in a micro polar fluid, Computers \& Fluids, 2011, 47, 1621

[15] Prakash J. and Sinha P., Squeeze film theory for micro polar fluids, ASME J. Lubrication Tech., 1976, 98(1), 139-144 .

[16] Papautsky. I. et al., Laminar fluid behaviour in micro channels using micro polar fluid theory, Sensors and Actuators, 1999, 73, 101-108

[17] Bhargava R., Sharma S., Bhargava P., Anwar Bég O. and Kadir A., Finite element simulation of nonlinear convective heat and mass transfer in a micropolar fluid-filled enclosure with Rayleigh number effects, Int. J. Applied Computational Mathematics, 2016, DOI: 10.1007/s40819-016-0180-9 (33pages)

[18] Latiff N. A., Uddin M. J., Anwar Bég O. and Ismail A. I. M., Unsteady forced bioconvection slip flow of a micropolar nanofluid from a stretching/ shrinking sheet, Proc. IMECHEPart N: J. Nanoengineering and Nanosystems, 2015, DOI: 10.1177/1740349915613817.

[19] M. S. Alam, Tarikul Islam and M. M. Rahman, Unsteady hydromagnetic forced convective heat transfer flow of a micropolar fluid along a porous wedge with convective surface boundary condition, International Journal of Heat and Technology 2015, 33(2), 115-122.

[20] Cheng C-Y., Free convection heat transfer from a nonisothermal permeable cone with suction and temperaturedependent viscosity, J. Applied Science and Engineering, 2015, $18,17-24$

[21] Solchana C., Ashwinkumar. G. P. and Sandeep. N., Magneto hydrodynamic flow towards a vertical rotating cone in porous medium with thermophoresis and Brownian motion effects, Int. J. Advanced Science and Technology, 2016, 86, 61-74

[22] Cheng C-Y., Natural convection heat transfer about a vertical cone embedded in a tridisperse porous media with constant wall temperature, Transport in porous media, 2015, 107, 765- 
[23] Yih K.A., Effect of radiation on natural convection about a truncated cone, Int. J. Heat Mass Transfer, 1999, 42, 4299-4305

[24] Gorla R. S. R., Schoren W. R. and Takhar H. S., Natural convection boundary layer flow of a micropolar fluid over an isothermal cone, Acta Mechanica, 1986, 61, 139-152

[25] Prasad V.R., Abdul Gaffar S., Keshava Reddy E. and Anwar Bég 0 ., Numerical study of non-Newtonian boundary layer flow of Jeffrey's fluid past a vertical porous plate in a non-Darcy porous medium, Int. J. Comp. Meth. Engineering Science \& Mechanics, 2014, 15(4) 372-389

[26] Subba Rao. A, V.R. Prasad, N. Nagendra, (2015) Heat Transfer in a non-Newtonian Jeffrey's fluid over a non-Isothermal Wedge, Procedia Engineering, Elsevier, 2015, 127C, 775-782; Doi: 10.1016/j.proeng.2015.11.412

[27] Tripathi D. and Anwar Bég O., Mathematical modelling of heat transfer effects on swallowing dynamics of viscoelastic flood bolus through the human oesophagus, Int. J. Thermal Sciences, 2013, 70, 41-53

[28] Hayat T., Ashraf M.B., Shezad S.A. and Bayomi N.N., Mixed convection flow of viscoelastic nanofluid over a stretching cylinder, J. Brazilian Society of Mechanical Sciences and Engineering, 2015, 37, 849-859

[29] Kodandapani M. and Srinivas S., Peristaltic transport of a Jeffery fluid under the effect of magnetic field in an asymmetric channel, Int. J. Nonlinear Mech., 2008, 43, 915-924

[30] Nadeem S. and Akbar N.S., Peristaltic flow of a Jeffrey's fluid with variable viscosity in an asymmetric channel, $Z$. Naturforsch A, 2009, 64A, 713-722

[31] Hayat T., Shehzad. S.A., Qasim M. and Obaidat. S., Radiative flow of Jeffery fluid in a porous medium with power law heat flux and heat source, Nuclear Engineering and Design, 2012, 243, 15-19

[32] Prasad V. R., Gaffar S. A. and Anwar Bég 0., Heat and mass transfer of a nanofluid from a horizontal cylinder to a micropolar fluid, AIAA J. Thermophysics Heat Transfer, 2015, 29, 1, 127-139

[33] Khadrawi, A.F., Al-Nimr. M.A. and Ali Othman, Basic viscoelastic fluid flow problems using the Jeffrey's model, Chemical Engineering Science, 2005, 60, 7131-7136

[34] Eringen A.C., Micro-continuum Field Theories: II- Fluent Media, Springer, New York, 2001

[35] Cheng C.Y., Natural convection of a micropolar fluid from a vertical truncated cone with power-law variation in surface temperature, Int. Comm. Heat Mass Transfer, 2008, 35, 39-46
[36] Anwar Bég O., Sandile S. Motsa, Kadir A., Tasveer A. Bég and Islam M.N., Spectral quasilinear numerical simulation of micropolar convective wall plumes in high permeability porous media, J. Engineering Thermophysics, 2016, 25(4), 1-24

[37] Malik M.Y. et al., MHD flow of tangent hyperbolic fluid over a stretching cylinder: using Keller box method, J. Magnetism and Magnetic Materials, 2015, 395, 271-276, Iranica, 20(3), 467-476, DOI: 10.1016/j.scient.2013.02.013

[38] Subba Rao. A, V. R. Prasad, V. Nagaradhika, O. Anwar Beg, Heat Transfer in Viscoplastic Boundary Layer flow from a vertical permeable Cone with momentum and thermal wall slip: Numerical study. Heat Transfer Research, 2018, 49(3), 189-204

[39] Gaffar S.A., Ramachandra Prasad. V, Keshava Reddy. E. and Anwar Bég 0., Numerical study of non-Newtonian Jeffrey's fluid from a permeable horizontal isothermal cylinder in non-Darcy porous medium, J of Brazilian Society of Mechanical Sciences and Engineering, 2015, 37, 1765-1783

[40] Subba Rao. A, V.R. Prasad, N. Bhaskar Reddy and O. Anwar Beg, Modelling Laminar Transport Phenomena In A Casson Rheological Fluid From An Isothermal Sphere With Partial Slip; Thermal science, 2015, 19(5), 1507-1519, doi:10.2298/TSCI120828098S

[41] A. Subba Rao, V. R Prasad , O. Anwar Beg and M. Rashidi, Free Convection Heat and Mass Transfer of a Nanofluid past a Horizontal Cylinder embedded in a non-Darcy porous medium; Journal of Porous Media, 2018, 21(3), 279-294

[42] Subba Rao. A, V.R. Prasad, N. Bhaskar Reddy, O. Anwar Beg, Modelling Laminar Transport Phenomena in a Casson rheological fluid from a Semi-Infinite Vertical Plate with Partial Slip; Heat Transfer-Asian Research, 2015, 44(3), 272-291, DOI: $10.1002 / \mathrm{htj} .21115$

[43] Subba Rao. A., Ramachandra Prasad V., Nagendra. N, Bhaskar Reddy. $\mathrm{N}$ and Anwar Beg O., Non-Similar computational solution for boundary layer flows of non-Newtonian fluid from an inclined plate with thermal slip, J. Applied Fluid Mechanics, 2016, 9, 795-807

[44] A. Subba Rao, CH. Amanulla, N. Nagendra, M. Surya Narayana Reddy and O. Anwar Beg, Hydro magnetic Non-Newtonian Nanofluid Transport Phenomena Past an Isothermal Vertical Cone with Partial Slip: Aerospace Nanomaterial Enrobing Simulation; Heat Transfer - Asian Research, 2018, 47(1), 203-230

[45] Keller H.B., Numerical methods in boundary-layer theory, Ann. Rev. Fluid Mech., 1978, 10, 417-433 